\title{
A Operational Risk Evaluation Research for Instrument Landing System
}

\author{
Zhang Rui ${ }^{1,}$, Fan Yong ${ }^{1, b}$, Cheng Rong ${ }^{1, c}$ \\ ${ }^{1}$ Guanghan Sub-college, Civil Aviation Flight University of China , Guanghan, Sichuan, 618307, \\ China \\ acafuczr@163.com, b ghfyfy@163.com csancer123@163.com
}

Keywords: Instrument Landing System, operational risk evaluation, SHEL model, analytic hierarchy process, fuzzy synthetic discrimination

\begin{abstract}
Instrument Landing System ILS is widely used in flight precision approach at present, therefore a operational risk evaluation system is in need of protecting the instrument safety operation in theory. This article adopts SHEL model for setting up a risk evaluation system and adopts analytic hierarchy process and fuzzy synthetic discrimination for analysis the system index in qualitative and quantitative, it can avoid one-sidedness. In the practical application, this evaluation system can assess the ILS objectively and accurately.
\end{abstract}

\section{Introduction}

With the rapid development of the China domestic flights and regional airports, the instrument landing system (ILS) occupy an important position in the protection of civil aviation security at present. But at present, risk evaluation studies for air traffic control equipment also achieve some results in China. In terms of qualitative risk evaluation of air traffic control facilities, document[1] were studied to determine the factors that affect the protection of air traffic control equipment operation, but failed to establish a reasonable evaluation model. document[2] proposed a fuzzy comprehensive evaluation of air traffic control equipment risk evaluation study, but failed to establish an appropriate evaluation model, so the evaluation of air traffic control equipment is more one-sided. document[3] from the quantitative aspects of the air traffic control equipment supported a capability evaluation model and established the index system, analysed and evaluated the factors that affect the ability of the device.

According to the basis and requirements of "civil air traffic management and safety management system (SMS) construction guidebook" [4], using qualitative and quantitative methods to model and calculate assignment this paper designs a specification model system for instrument landing system operational risk evaluation, determines the weight of each index, adopts the fuzzy comprehensive evaluation to complete a risk evaluation and quantitative results. According to this study., this paper can actually provide theoretical support for the instrument landing system for risk evaluation and hazard identification.

\section{ILS operational risk evaluation model design and algorithm}

Risk evaluation model and index design. According ILS failure statistics show ILS safe operation could be affect by many factors, these factors are interrelated and influence each other, in accordance with the requirements of the "civil aviation air traffic management and safety management system (SMS) construction Guidebook", "hazard identification should be from people, 
machines, route, management and other aspects, using various methods and tools to analyze the various factors that may affect safety."[4]. Therefore, the design of ILS risk evaluation index model must be based on the principle of systematic, objectivity, development, feasibility and independence [5].

In accordance with the above requirements, using SHEL model [6] on the ILS risk evaluation model for design, people (live ware), software (software), the environment (environment) and at the center of the hardware (hardware) between relation are needed to take into account in the entire system, the relationships between the interface of them are:

Hardware and human (H-L) are needed a consideration that is the relationship between people and equipment, namely the ability of people to operate the equipment and so on.

Hardware and software (H-S) contain the current manual system, whether it meets regulatory and other equipment.

Hardware and Environment (H-E) contain the environment, climate, whether they meets the operational conditions of the equipment, etc.

Hardware and Hardware $(\mathrm{H}-\mathrm{H})$ contains whether the affiliated equipment is able to support the current equipment operation, the spare parts and other equipment is available.

Based on the above analysis ILS risk evaluation model, the model is divided into three layers: goal, criterion and target layer (refer with: Fig.1), covering the main factors ILS operational risk evaluation.

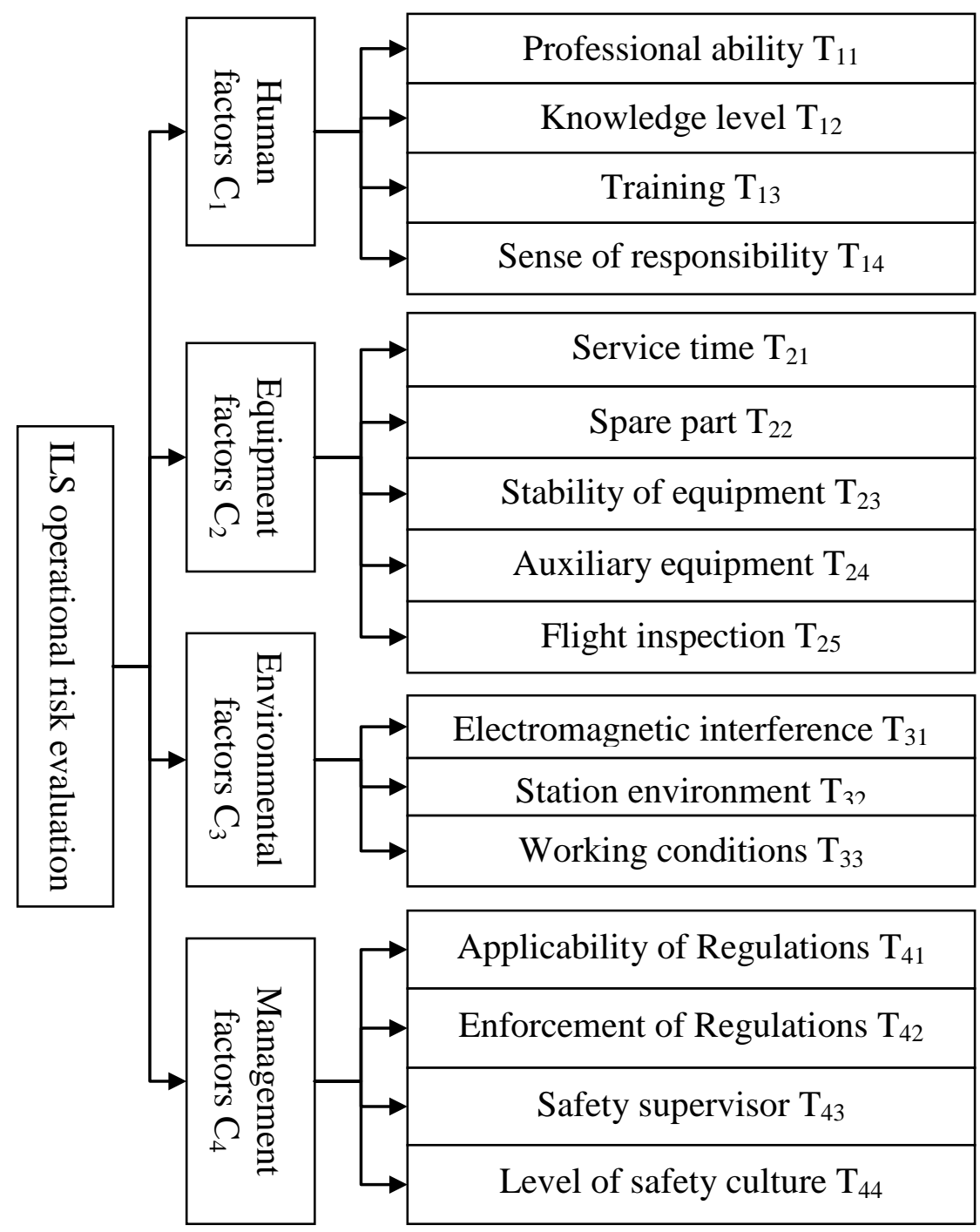

Fig.1 Operational risk evaluation system for ILS 
Operational risk evaluation algorithms and index calculation. Before assessing ILS risk evaluation model, need to calculate the target and criterion layers index weight. The process of ILS operational risk evaluation is a fuzzy complex, but fuzzy comprehensive evaluation method can be assess quantify things which is hard to describe , therefore the use of the analytic hierarchy process and fuzzy comprehensive evaluation [7] can assess ILS risk evaluation algorithm , as follows:

(1)Establish factors collection. According to the evaluation model, establish the criterion and target layers index:

Criterion layer index is: $G=\left(C_{1}, C_{2}, \ldots, C_{\mathrm{m}}\right)$,

Target layer index is: $C_{i}=\left(T_{i 1}, T_{i 2}, \ldots, T_{i n}\right), i=1,2, \ldots, m$

(2)Set up the index weight collection of the criterion and target layers. Each factors collection's importance is determined by index weight which is defined by mathematical statistics method and nine marks method[8], normalized and last step is verify compliance.

The criterion layer index weight is: $W=\left(W_{1}, W_{2}, \ldots, W_{m}\right)$ and $\sum_{\mathrm{k}=1}^{\mathrm{m}} \mathrm{W}_{\mathrm{k}}=1$

Target layer index weight is: $\mathrm{W}_{i}=\left(w_{i 1}, w_{i 2}, \ldots, w_{i n}\right), i=1,2, \ldots, m$ and $\sum_{k=1}^{n} w_{i k}=1$

(3)Set up evaluation collection. Evaluate collection is the result of evaluation system. $V=\left(v_{1}, v_{2}, \ldots, v_{i}\right)$.

As the above, $v_{i}$ means all kinds of evaluation collection, the evaluation collection establish a corresponding vector and generate $v_{i}$ data one by one, the vector is: $\bar{V}=\left(\bar{v}_{1}, \bar{v}_{2}, \ldots, \bar{v}_{i}\right)$.

(4)Set up single factor evaluation matrix by criterion layer. Each index are graded by Experts in different kinds of filed in ATC, gotten a statistics and normalization by evaluation collection, finally can get the frequency evaluation matrix $F_{i}$ of criterion layer: $F_{i}=\left[\begin{array}{cccc}f_{i 11} & f_{i 12} & \cdots & f_{i 1 m} \\ f_{i 21} & f_{i 22} & \cdots & f_{i 2 m} \\ \vdots & \vdots & \vdots & \vdots \\ f_{i n 1} & f_{i n 2} & \cdots & f_{\text {ink }}\end{array}\right]$

Weight $W_{i}$ and $F_{i}$ can calculate the fuzzy relation matrix $R_{i}: R_{i}=W_{i} \times F_{i}$

(5)Repeat the step(4) by target layers, it can get all of the criterion layer evaluation matrix $\left(R_{1}, R_{2}, \ldots, R_{i}\right)^{T}$, then the fuzzy relation matrix of evaluation system is: $R=\left(W_{1}, W_{2}, \ldots W_{n}\right) \times\left(R_{1}, R_{2}, \ldots, R_{n}\right)^{T}$

The finally result of risk evaluation $\mathrm{S}$ is: $S=R \times \bar{V}$

Computational examples. Take ILS in Guanghan airport for example and choose 10 experts in ATC filed, they grade the ILS risk model by nine marks method, get the target layer $t_{i}$ matrix, criterion layer $\mathrm{c}_{\mathrm{i}}$ matrix and goal layer judgment matrix(see table 1,table 2, table 3,table 4, table 5). 
Table $1 \mathrm{C}_{1}-\mathrm{T}_{1 \mathrm{k}}$ judgment matrix

\begin{tabular}{ccccc}
\hline$C_{1}$ & $T_{11}$ & $T_{12}$ & $T_{13}$ & $T_{14}$ \\
\hline$T_{11}$ & 1 & 3 & 2 & 3 \\
$T_{12}$ & $1 / 3$ & 1 & $1 / 2$ & 1 \\
$T_{13}$ & $1 / 2$ & 2 & 1 & 2 \\
$T_{14}$ & $1 / 3$ & 1 & $1 / 2$ & 1 \\
\hline
\end{tabular}

Table $3 \mathrm{C}_{3}-\mathrm{T}_{3 \mathrm{k}}$ judgment matrix

\begin{tabular}{cccc}
\hline$C_{3}$ & $T_{31}$ & $T_{32}$ & $T_{33}$ \\
\hline$T_{31}$ & 1 & $1 / 2$ & 2 \\
$T_{32}$ & 2 & 1 & 2 \\
$T_{33}$ & $1 / 2$ & $1 / 2$ & 1 \\
\hline
\end{tabular}

Table 5 G-C i $_{\text {judgment matrix }}$

\begin{tabular}{ccccc}
\hline$G$ & $C_{1}$ & $C_{2}$ & $C_{3}$ & $C_{4}$ \\
\hline$C_{1}$ & 1 & 2 & 3 & 2 \\
$C_{2}$ & $1 / 2$ & 1 & 2 & 1 \\
$C_{3}$ & $1 / 3$ & $1 / 2$ & 1 & $1 / 2$ \\
$C_{4}$ & $1 / 2$ & 1 & 2 & 1 \\
\hline
\end{tabular}

Take matrix $\mathrm{C}_{1}-\mathrm{T}_{1 \mathrm{k}}$ for example, the Eigenvector of importance judgment matrix $\mathrm{r}$ is:

$r=(0.8099,0.2505,0.4674,0.2505)$

Normalized and get the weight vector:

$$
W_{1}=(0.4554,0.1409,0.2628,0.1409)
$$

Repeat the above steps and calculate other target layer and criterion layer, can get the weight vector one by one:

$$
\begin{aligned}
& W_{2}=(0.0972,0.1598,0.2621,0.0637,0.4172) \\
& W_{3}=(0.3108,0.4934,0.1958) \\
& W_{4}=(0.1409,0.1409,0.4554,0.2628) \\
& W=(0.4236,0.2271,0.1223,0.227)
\end{aligned}
$$

Set five factors evaluation collection $V=$ (serious, relatively serious, common, slight, good ) and corresponding quantitative vector $\bar{V}=(15,45,65,85,95)$. Through 10 experts grade all the factor of target layer, get the Indexes of frequency distribution(See table 6).

Normalized the target layer frequency, using algorithm(4),can get human factors fuzzy evaluation matrix $R_{1}$ :

$$
R_{1}=W_{1} \times F_{1}=(0,0,0.5507,0.4230,0.0263)
$$

The equipment , environment and management factors fuzzy evaluation matrix $R_{2}, R_{3}$ and $R_{4}$ is: 


$$
\begin{aligned}
& R_{2}=(0,0.1348,0.3602,0.3318,0.1733) \\
& R_{3}=(0,0.0493,0.3676,0.5520,0.0311) \\
& R_{4}=(0,0.0227,0.4834,0.4939,0)
\end{aligned}
$$

At last, throught $R_{1}, R_{2}, R_{3}$ and $R_{4}$, ILS operation risk evaluation matrix $R$ and index $S$ is

$$
\begin{aligned}
& R=W \times\left(R_{1}, R_{2}, R_{3}, R_{4}\right)^{T}=(0,0.0418,0.4698,0.4342,0.0543) \\
& S=R \times \bar{V}=(0,0.0418,0.4698,0.4342,0.0543) \times(15,45,65,85,95)^{T}=74.48 \text { ILS risk index is }
\end{aligned}
$$

\begin{tabular}{|c|c|c|c|c|c|}
\hline & serious & $\begin{array}{c}\text { relatively } \\
\text { serious }\end{array}$ & common & slight & good \\
\hline$T_{11}$ & 0 & 0 & 7 & 3 & 0 \\
\hline$T_{12}$ & 0 & 0 & 4 & 6 & 0 \\
\hline$T_{13}$ & 0 & 0 & 4 & 5 & 1 \\
\hline$T_{14}$ & 0 & 0 & 5 & 5 & 0 \\
\hline$T_{21}$ & 0 & 4 & 6 & 0 & 0 \\
\hline$T_{22}$ & 0 & 6 & 4 & 0 & 0 \\
\hline$T_{23}$ & 0 & 0 & 7 & 3 & 0 \\
\hline$T_{24}$ & 0 & 0 & 2 & 7 & 1 \\
\hline$T_{25}$ & 0 & 0 & 1 & 5 & 4 \\
\hline$T_{31}$ & 0 & 0 & 2 & 7 & 1 \\
\hline$T_{32}$ & 0 & 1 & 5 & 4 & 0 \\
\hline$T_{33}$ & 0 & 0 & 2 & 8 & 0 \\
\hline$T_{41}$ & 0 & 0 & 3 & 7 & 0 \\
\hline$T_{42}$ & 0 & 1 & 6 & 3 & 0 \\
\hline$T_{43}$ & 0 & 0 & 5 & 5 & 0 \\
\hline$T_{44}$ & 0 & 0 & 7 & 3 & 0 \\
\hline
\end{tabular}

between "common" and "slight”, according to the membership, ILS operation risk "common” is 0.4698,"slight” is 0.4342 , therefore there is a demand that improve security.

\section{Conclusions}

At present, there is no uniform standard evaluation index and method. This paper uses SHEL model to set up evaluation index system, adopts fuzzy evaluation and analytic hierarchy process to analyze index that could be more perfect and accurately, finds problems and solves problem in the process, makes ILS operating security.

\section{References}

[1] Yuan G H. Talk about ATC equipment risk evaluation management[J]. Air traffic management, 2009,8:39-41.

[2] Cai H B.Based on multi-layers fuzzy evaluation in ATC equipment risk evaluation[J]. Information \& communication,2013,139(7):4-5. 
[3] Zhang X W,Tao Y. Base on fuzzy comprehensive evaluation in ATC equipment support capability evaluate[J]. Value engineering,2012,14:5-26.

[4] CAAC ATC management office. civil air traffic management and safety management system (SMS) construction guidebook[EB], 2011-05-10 $)$ [2014-06-23].http://www.caac.gov.cn/B1/GLCX/201105/P020110511304138875124.pdf.

[5] Luo F,Huang R R,Wang H J ,etal.Research on evaluation index system for communication risk between controllers and pilots in air traffic[J].China Safety Science Journal, 2010,20(5):162-165.

[6] Li H F,Zhang W H, Wu X P. The Human factor engineering application on civil aviation incidents analysis[J]. Chinese Journal of Ergonomics, 2009,15(1):56-59.

[7] Du D, Pang Q H, Wu Y. Modern evaluation methods and Example [M].Beijing:TsingHua University press,2008:4-10.

[8] $\mathrm{Xu} \mathrm{J,} \mathrm{Liu} \mathrm{N.} \mathrm{The} \mathrm{basic} \mathrm{idea} \mathrm{and} \mathrm{practical} \mathrm{applications} \mathrm{of} \mathrm{analytic} \mathrm{hierarchy} \mathrm{process[J].}$ Information Research, 2008,134(12):113-115. 